\title{
Lipodystrophy in HIV patients: its challenges and management approaches
}

\author{
This article was published in the following Dove Press journal: \\ HIVIAIDS - Research and Palliative Care \\ I3 December 2011 \\ Number of times this article has been viewed
}

\section{Rohit Singhania \\ Donald P Kotler}

Department of Medicine, St Luke'sRoosevelt Hospital Center, New York, NY, USA

Correspondence: Donald P Kotler Gastrointestinal Division, S\&R I2, St Luke's-Roosevelt Hospital Center, I I I Amsterdam Avenue, New York, NY 10025, USA

$\mathrm{Tel}+\mathrm{I} 2$ I 25233670

Fax + I 2125233678

Email dkotler@chpnet.org
Abstract: HIV-associated lipodystrophy is a term used to describe a constellation of body composition (lipoatrophy and lipohypertrophy) and metabolic (dyslipidemia and insulin resistance) alterations that accompany highly active antiretroviral therapy. These changes, which resemble metabolic syndrome, have been associated with a variety of adverse outcomes including accelerated cardiovascular disease. The body composition and metabolic changes appear to cluster in HIV infection, although they are distinct alterations and do not necessarily coexist. Epidemiological studies have demonstrated multiple pathogenic influences associated with host, disease, and treatment-related factors. The adverse treatment effects were more prominent in early regimens; continued drug development has led to the application of metabolically safer regimens with equal or greater potency than the regimens being replaced. Disease-related factors include HIV infection as well as inflammation, immune activation, and immune depletion. The body composition changes promote anxiety and depression in patients and may affect treatment adherence. Treatment of dyslipidemia and alterations in glucose metabolism is the same as in non-HIV-infected individuals. Lipoatrophy is managed by strategic choice of antivirals or by antiviral switching, and in some cases by plastic/reconstructive surgery. Lipohypertrophy has been managed mainly by lifestyle modification, ie, a hypocaloric diet and increased exercise. A growth hormone releasing factor, which reduces central fat, has recently become available for clinical use.

Keywords: lipoatrophy, lipohypertrophy, body composition, dyslipidemia, insulin resistance

\section{Introduction}

The term lipodystrophy is used to describe a diverse group of disorders characterized by a constellation of body composition and metabolic alterations. ${ }^{1}$ Changes in body composition include lipoatrophy, which is complete or partial loss of adipose tissue compartments, and lipohypertrophy, which is a pathological accumulation of adipose tissue in distinct body compartments. Lipoatrophy and lipohypertrophy may or may not coexist. Metabolic abnormalities include insulin resistance and dyslipidemia. Hepatic steatosis may also be recognized. The severity of these comorbidities usually correlates with the degree of adipose tissue loss. Accompanying complications may include hypertension, proteinuric kidney disease, acanthosis nigricans, and polycystic ovary syndrome.

Lipodystrophy may be inherited or acquired; the former is rarely seen (Table 1). By far, the most common type of lipodystrophy seen in the last 2 decades is an acquired form that occurs in HIV-infected individuals treated with highly active antiretroviral therapy (HAART), where reported prevalence rates range from a few percent to over $80 \% .^{2}$ A major confounding factor in this field has been uncertainty as to whether 
Table I Types of lipodystrophy

Acquired
Acquired generalized lipodystrophy
Acquired partial lipodystrophy
HIV-associated lipodystrophy
Localized lipodystrophy
Inherited
Congenital generalized lipodystrophy
Type I
Type 2
Familial partial lipodystrophy
Dunnigan variety
PPAR- $\gamma$ mutations
Mandibuloacral dysplasia
Type A lipodystrophy
Type B lipodystrophy

Note: Summarized from Garg.'

Abbreviation: PPAR- $\gamma$, peroxisome proliferator-activated receptor gamma.

the body composition and metabolic alterations represent a single syndrome or a variety of coexisting abnormalities, or even if some of the alterations are related to the development of metabolic syndrome. For the purpose of this article, the body composition and metabolic alterations will be grouped together and referred to as "lipodystrophy."

With the recognition that HIV production could be suppressed but not eradicated, the focus of therapy in the mid-1990s was to convert the infection from a uniformly fatal disease into a long-term, manageable condition. This accomplishment was reported in 1998, with the seminal publication from the Multicenter AIDS Cohort Study (MACS) associating a dramatic drop in death rate from AIDS with widespread application of HAART in the United States. ${ }^{3}$ However, the frequent occurrence of diabetes mellitus, insulin resistance, dyslipidemia, lipoatrophy, central lipohypertrophy accumulation, ${ }^{4}$ and premature cardiovascular disease was also reported at the time. As excess morbidity and mortality from these complications increase the risks and decrease the benefits of HAART, vigorous efforts have been made to define the metabolic and other health risks associated with HIV infection and HAART, as well as to develop safer agents and treatment regimens.

\section{Clinical presentation Body composition}

The clinical diagnosis of lipodystrophy is usually made on the basis of physical examination, by recognizing changes in the sizes of different body fat compartments, especially the presence of lipoatrophy. Lipoatrophy is seen as a loss of subcutaneous fat in the extremities or face. Loss of fat in the lower extremities may lead to an appearance of prominent veins in the legs, reaching to the upper thighs, while fat loss in the face is especially prominent in the cheeks. Early in the HAART era, it was often difficult to distinguish fat loss from lipoatrophy or from wasting related to protein-energy malnutrition. One key difference is that lean mass and skeletal muscle mass are normal in patients with lipodystrophy but are low in malnourished patients. While obvious in full-blown cases, inspection lacks diagnostic sensitivity. It has been estimated that a loss of around one-third of subcutaneous fat is needed before lipoatrophy becomes clinically apparent. ${ }^{5}$ The required losses may be even higher in patients who are obese at treatment initiation.

Although the diagnosis does not depend on quantitating the degree of adipose tissue loss, estimations can be made in clinical settings. The most practical and cost-effective, and hence frequently used, modalities are anthropometric measurements of biceps, triceps, and subscapular skin folds, as well as hip and waist circumferences. More accurate methods, which offer superior objectivity and precision, are dual energy X-ray absorptiometry and the cross-sectional imaging techniques, magnetic resonance imaging (MRI) and computerized tomography (Figure 1). These techniques are limited to research settings due to high costs and to lack of third-party insurance coverage, availability for everyday clinical practice, and generally accepted normal ranges and definitions of abnormality. Ultrasonography is another potential alternative to estimate intra-abdominal fat content; it has accuracy and precision and yet is affordable as well as accessible.

A gain in truncal and upper body fat content (specifically, visceral fat content) may be seen in treated patients in the presence or absence of lipoatrophy. In women, the increase in fat may include the breasts. The health effects of truncal obesity are well known to clinical medicine. Jean Vague, ${ }^{6}$ a French investigator, defined two distinct morphologic obesity subtypes in women: android or upper body obesity, which was associated with insulin resistance, diabetes, and cardiovascular disease, and gynecoid or lower body obesity, which was not associated with these adverse outcomes. The key observation was that the differences in outcome were independent of actual fat mass, so that it was the body distribution of fat and not the obesity per se that predicted poor outcomes.

The interrelationships between the changes in the sizes of subcutaneous and visceral fat compartments were initially uncertain. Data from the Fat Redistribution and Metabolic Change in HIV Infection (FRAM) study, which compared the results of body composition and metabolic measurements 

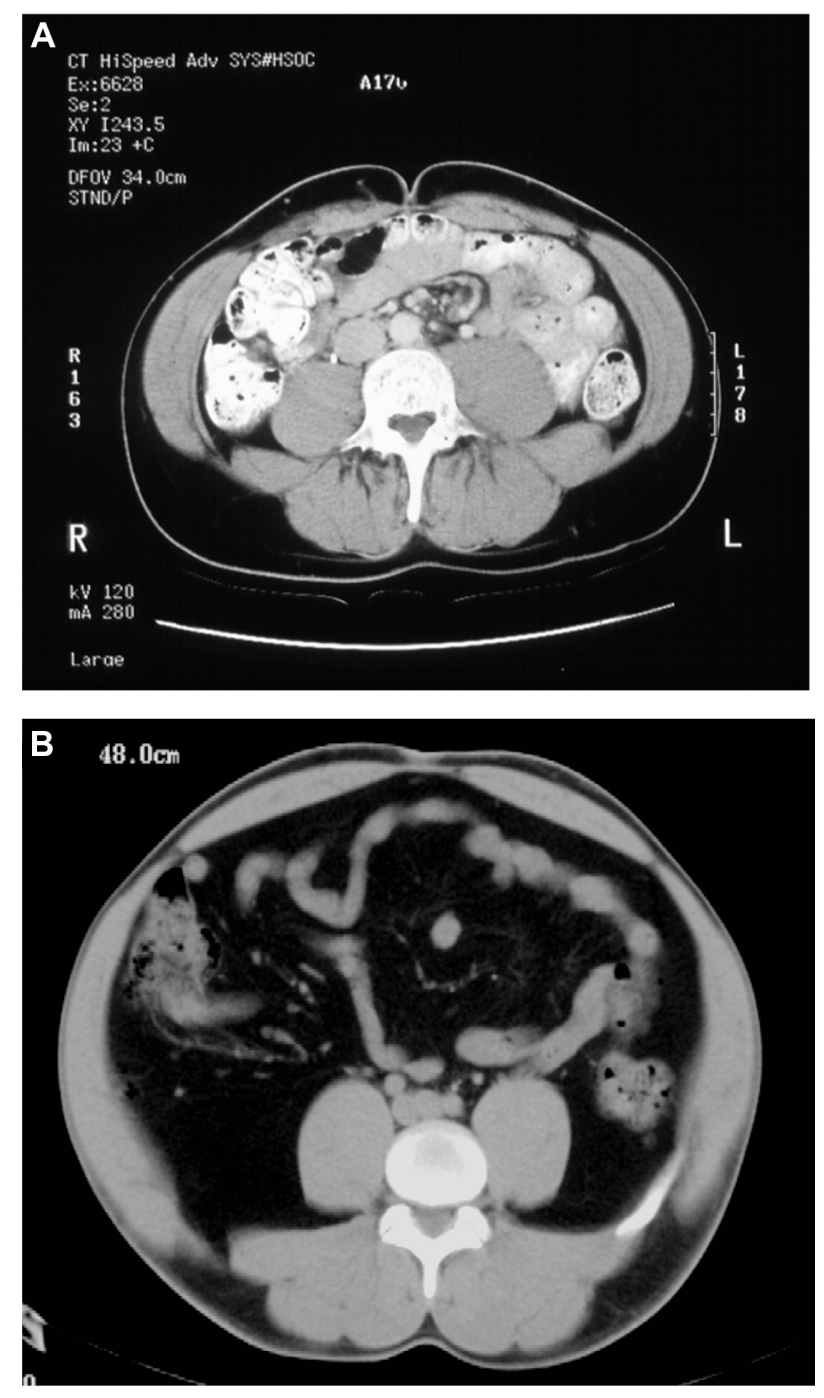

Figure I Computerized tomography scans of $(\mathbf{A})$ a healthy adult and $(\mathbf{B})$ a patient with coexisting lipoatrophy and lipohypertrophy. In comparison with the healthy adult, the patient with coexisting lipoatrophy and lipohypertrophy has less subcutaneous adipose tissue and an increase in intra-abdominal (visceral) adipose tissue.

in HIV- and non-HIV-infected subjects, showed that HIVinfected men who reported a loss of body fat had significantly less subcutaneous adipose tissue (SAT), as measured by whole-body MRI, than non-HIV-infected controls. ${ }^{7}$ However, HIV-infected men who did not report a loss of body fat also had significantly less SAT than did controls, so that lipoatrophy was associated with HIV infection. The differences in women were smaller but were in the same direction. ${ }^{8}$ In contrast to the SAT results, the results of visceral adipose tissue (VAT) measurements showed that mean values were lower in the HIV-infected group than in the controls. These results differed from the expectation that the loss in SAT in the HIV group would be associated with a gain in VAT (ie, fat redistribution). It should be noted that the results of this cross-sectional study do not point to a cause for the difference (ie, viral infection versus antiretroviral agents, or other factors). In a later, longitudinal analysis of the MACS cohort, investigators showed a similar gain in waist circumference in both HIV-infected and control men. ${ }^{9}$ Thus, it appears that an increase in truncal fat content is relatively common but is not necessarily related to HIV infection or to its treatment.

\section{Metabolic alterations}

Metabolic alterations - namely, dyslipidemia and insulin resistance - frequently accompany HAART therapy and have become a widespread concern due to their link to cardiovascular disease. Elevated triglyceride levels are seen most commonly with the use of protease inhibitor (PI) therapy. Mild hypercholesterolemia is observed in 30\%-50\% of patients. However, dyslipidemia was seen commonly in the pre-antiretroviral era. ${ }^{10}$ In the pre-HAART era, increased triglyceride levels were associated with increased interferon alpha concentrations in HIV patients. ${ }^{11}$ While abnormalities in glucose metabolism were an uncommon complications of PI therapy in initial clinical trials $(2 \%-5 \%)$, the US Food and Drug Administration (FDA) reported the onset of diabetes in patients treated with PI therapy in $1997 .^{12}$

\section{Pathogenic mechanisms}

PIs were initially implicated as the primary cause of the observed changes. ${ }^{4}$ Direct effects of PIs on serum lipids and glucose metabolism were shown in non-HIV-infected volunteers. ${ }^{13,14}$ However, it soon was recognized that the detection of lipoatrophy preceded the application of PI therapy. ${ }^{15}$ Thymidine analog nucleoside reverse transcriptase inhibitors (NRTIs) were shown to promote lipoatrophy as well as metabolic alterations. In addition, non-nucleoside reverse transcriptase inhibitors (NNRTIs) have been shown to affect serum lipid concentrations.

The results of epidemiological studies performed since the late 1990s suggested the presence of multiple pathogenic mechanisms, including disease, host, and treatment-related influences (Table 2). Importantly, there were distinctions in the relative importance of various factors as well as much overlap, suggesting that lipodystrophy is not a single process but rather a condition whose expression is of multifactorial origin. The FRAM study has been particularly instrumental in demonstrating distinctions among its various components. While some continue to classify the condition as a single disease, most investigators and treating physicians now believe that the interrelationships among the body composition and metabolic alterations are loose and that the abnormalities can be evaluated and managed individually. 
Table 2 HIV-associated lipodystrophy: epidemiological associations

Host
Age
Eex
Individual genetic susceptibility
BMI
Diet
Exercise
Comorbidities
Therapy
Specific agent
Duration
Disease
Duration
Severity of immune depletion
Magnitude of immune reconstitution

Abbreviation: BMI, body mass index.

Another major uncertainty is the relative importance of the various influences. In general, the effects of specific antiviral agents in promoting body composition and metabolic alterations have diminished over the past 5-7 years as drug development has led to the application of less-toxic regimens. In addition, the effects of inflammation and immune activation related to the underlying viral infections (eg, HIV, hepatitis $\mathrm{C}$, cytomegalovirus, and so forth) have become more apparent, even in patients being successfully treated with HAART. The role of individual host susceptibilities, especially the role of specific genetic polymorphisms, is also increasingly recognized.

As noted, certain metabolic alterations such as hypertriglyceridemia were seen in the pre-HAART era, and even in the era prior to the development of antiviral therapies. ${ }^{10}$ An important observation was made by investigators of the MACS cohort, who had the opportunity to follow approximately 50 individuals from a period prior to HIV seroconversion through initiation and follow-up of HAART therapy. ${ }^{16} \mathrm{~A}$ fall in total, high-density lipoprotein (HDL), and low-density lipoprotein (LDL) cholesterol concentrations was noted after seroconversion and persisted as immune function declined. Initiation of HAART led to increases in total and LDL cholesterol, but only to near preinfection values for LDL cholesterol. The data were interpreted to show that the rise in serum LDL cholesterol concentration was related to reversal of an effect of infection in lowering serum lipids. Thus, a mild to moderate rise in total or LDL cholesterol concentration after HAART initiation may be viewed as a return to prior health, rather than as evidence of drug toxicity. However, if dyslipidemia preceded HIV infection, the return would be to a dyslipidemic condition. The same may be said of insulin resistance, truncal obesity, or other aspects of health and disease.

There is both clinical and experimental evidence for direct metabolic effects of antiretroviral agents. Some PIs reversibly inhibit glucose uptake into muscle and adipose tissue cells at the level of the glucose transport molecule GLUT4. ${ }^{17}$ PIs also may affect hepatic very-low-density lipoprotein secretion through an inhibition of intracellular apoprotein B degradation. ${ }^{18}$

NRTIs, especially thymidine analogs, may also have direct toxicity. Fialuridine, the first agent tested in chronic hepatitis B infection was the precedent for NRTI toxicity. The drug was virologically effective but it led to a high incidence of subacute liver failure with symptomatic lactic acidosis. ${ }^{19}$ The toxic effect was found to be mitochondrial toxicity related to inhibition of the effect of DNA polymerase $\gamma$, the DNA polymerase involved in mitochondrial function. Many clinical studies have linked NRTI therapy with mitochondrial toxicity, including lactic acidosis in HIV infection. The major cause of lipoatrophy was shown to be thymidine analog NRTI therapy, based on studies of antiretroviral-naïve subjects on their initial HAART regimens. ${ }^{20,21}$ Mitochondrial dysfunction also promotes insulin resistance and dyslipidemia. ${ }^{22,23}$ Depletion of mitochondrial DNA has been shown in both in vitro and in vivo studies, and partial reversal both of the mitochondrial DNA depletion and of clinical toxicity has been demonstrated after switching to alternative antiviral agents. $^{24}$

NNRTIs promote apoprotein A1 synthesis, which is associated with increased HDL cholesterol concentration. ${ }^{25}$ On the other hand, entry and integrase inhibitors appear to be free of metabolic toxicities.

Many studies have demonstrated genetic predispositions to development of body composition and metabolic abnormalities. The potential for genetically based differential susceptibility to NRTI toxicity was demonstrated by the observation that lactic acidosis in patients receiving thymidine analog NRTI therapy occurred mainly in AfricanAmerican women, even though the relative size of the treatment population at the time was reasonably low.

A single nucleotide polymorphism in the resistin gene was shown to be associated with a cluster of metabolic alterations including insulin resistance, dyslipidemia, and lipoatrophy in antiretroviral-naïve patients receiving an initial HAART regimen. ${ }^{26}$ Of note, pretreatment serum lipid and insulin concentrations were higher in the subgroup that developed body composition and metabolic alterations than 
in those that did not. Several other polymorphisms have been shown to affect the serum lipid response to antiviral therapy. Based on these data, it is likely that the development of severe metabolic alterations is related to an interaction among the viral infection, antiviral medications, and underlying predisposition to metabolic alterations. Many other single-nucleotide polymorphisms have been linked to various elements of lipodystrophy, including genes related to apoproteins, lipid metabolism, proinflammatory cytokines, drug efflux transport, and apoptosis. Mitochondrial haplotypes also may influence the susceptibility to developing lipodystrophy as they do in NRTI-associated neuropathy.

Several investigators have noted similarities between HIV-associated lipodystrophy and metabolic syndrome as seen in non-HIV-infected individuals, though some studies have shown that HIV-infected patients are more likely to have hypertriglyceridemia and are less likely to be hypertensive. In non-HIV-infected patients with metabolic syndrome, there is now substantial evidence linking inflammation and atherosclerosis. ${ }^{27}$ Many studies have documented persisting inflammation and immune activation in HIV infection, even in patients on suppressive HAART therapy. ${ }^{28}$ Chronic inflammation and immune activation are believed to play a role in long-term adverse outcomes, including mortality, cardiovascular disease, neurocognitive decline, osteopenia, and frailty, among others.

The relative roles of viral infection and its treatment in the genesis of cardiovascular disease in HIV infection were illustrated by the results of the Strategies for Management of Antiretroviral Therapy (SMART) study ${ }^{29}$ This was a seminal study that directly compared the effects of continuous viral suppression with HAART and with limiting the exposure to antiviral therapy by starting and stopping therapy based upon CD4 count. At the time the study was conceived, many believed that limiting antiviral exposure would decrease adverse outcomes, but the opposite was observed. Not only was symptomatic cardiovascular disease more common with intermittent treatment but also viral rebound was directly associated with several relevant alterations, including elevations in pro-inflammatory cytokines, coagulation factors, and decreased HDL cholesterol concentrations in this and another similarly designed study. ${ }^{30,31}$

The role of the underlying viral infection in promoting cardiovascular disease was corroborated in the AIDS Clinical Trials Group (ACTG) study 5152s, a metabolic substudy of ACTG 5142, which compared class-sparing regimens in antiretroviral-naïve subjects. ${ }^{32}$ Flow-mediated dilatation, a measure of endothelial function, was measured at baseline and after 12 weeks. Baseline values were abnormally low and rose moderately on treatment. Importantly, values rose similarly in all arms of the trial, and changes in endothelial function correlated with changes in plasma viral load, rather than any specific antiviral agent (Figure 2).

\section{Clinical significance}

The identification of lipodystrophy has had profound implications on the management of HIV. Its recognition as a side effect of HAART led to a re-evaluation of the appropriate time to start therapy (specifically, delaying initiation of HAART) and also to various modifications in therapies for HIV - namely, switching therapies, stopping therapy, and intermittent treatment all had in their rationale, at least in part, the desire to minimize drug exposure so as to avoid metabolic alterations and associated adverse outcomes including cardiovascular disease.

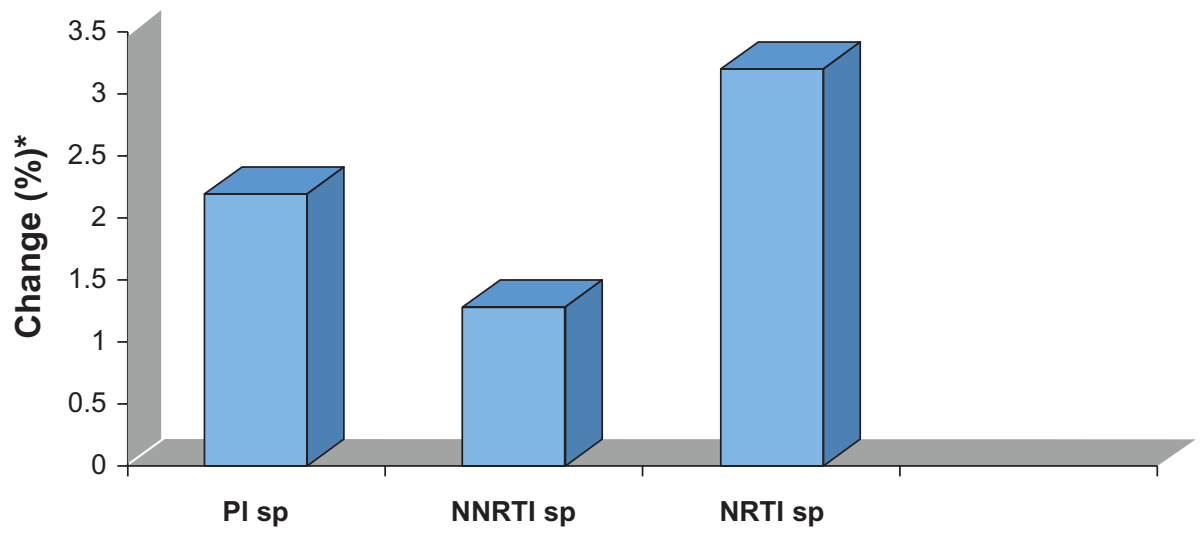

Figure 2 Change in endothelial function in the AIDS Clinical Trials Group study $5142 .{ }^{32}$ This study examined a class-sparing strategy and a substudy examined endothelial function as flow-mediated dilatation (FMD). FMD was impaired at baseline and improved during highly active antiretroviral therapy. The improvement was independent of a specific regimen and bore no relationship to baseline viral load; however, it had a significant association with the decrease in viral load.

Notes: Data as change in flow-mediated diameter, expressed as percent. Within-group differences: $P<0.005$ for PI-sp and NNRTI-sp regimens; $P=0.015$ for NRTI-sp regimen. "Data expressed as percent and presented as absolute change compared with pretreatment values.

Abbreviations: NNRTI, non-nucleoside reverse transcriptase inhibitor; NRTI, nucleoside reverse transcriptase inhibitor; PI, protease inhibitor; sp, sparing. 
Various quality-of-life studies in HIV lipodystrophy have generally shown that patients enjoy a good quality of life, including good physical functioning, whereas common complaints manifesting as anxiety and depression are related mostly to the stigma associated with morphologic changes. ${ }^{33,34}$ HIV-infected patients may refuse therapy or reduce treatment compliance as a result of or in order to avoid these side effects. As a result, the patient may experience rebound in viral load or the development of viral resistance. This will eventually lead to disease progression and even to an increased risk of disease transmission, possibly by a drug-resistant viral strain.

The changes associated with lipodystrophy (body fat redistribution, dyslipidemia, and insulin resistance) are associated with cardiovascular disease. Many cohort studies have implicated an increased risk for developing symptomatic cardiovascular disease. ${ }^{35,36}$ However, the relative risk of developing symptomatic cardiovascular disease is uncertain since these studies lack internal control groups. Analysis of the D:A:D cohort suggests that the Framingham equation for cardiovascular risk modestly underestimates the actual risk in HIV-infected individuals. ${ }^{37}$ That being said, the benefits of HAART therapy far outweigh the cardiovascular risk in the form of reduced risk of illness and death, and antiviral therapy certainly should not be withheld for fear of promoting cardiovascular disease.

\section{Diagnosis}

There has been considerable debate about the appropriate diagnostic criteria for this syndrome, but little consensus has been reached. A multifactorial equation, the Lipodystrophy Severity Grading Scale, was developed to discriminate between patients generally agreed to have and patients generally agreed not to have the syndrome, and has the potential to act as a tool for case definition..$^{38}$ However, this equation requires extensive evaluation using technologies not easily accessed clinically, and it is not helpful therapeutically, since therapies are based upon the specific alterations rather than lipodystrophy per se. Other scales have been developed for assessing subcutaneous lipoatrophy, facial lipoatrophy, and central fat accumulation, as well as quality of life, anxiety, and depression. These scales have been applied more in research than in clinical management. As a consequence, practitioners of HIV medicine have become familiar with practice standards for internal medicine in addition to those related specifically to the management of HIV infection.

Several sets of guidelines for metabolic alterations have been published. ${ }^{39,40}$ In general, the recommendations parallel those developed for similar metabolic alterations in the absence of HIV infection.

\section{Therapeutic options}

There are five basic possible strategies to manage the body composition and/or metabolic changes: (1) avoid by strategic choice of antiviral agents; (2) do not intervene; (3) stop HAART; (4) switch HAART; and (5) treat the individual abnormalities while continuing HAART. For some practitioners, viral suppression is of paramount importance; clinicians treat the various abnormalities individually and do not change an effective antiviral regimen. Others believe that the metabolic alterations are dangerous. In the past, clinicians have sometimes discontinued therapy completely; presently, switching to an alternative antiviral regimen, if there is one available, is more common.

The notion of treatment in lipodystrophy is complicated. Treatment includes both prevention and corrective therapy. It is important to define the specific outcomes, which range from asymptomatic changes in serum lipid concentrations to fatal cardiovascular disease, when analyzing specific treatments. In addition, the effect sizes of the various treatments vary considerably.

The overall risks and benefits of HAART in relation to the outcome of cardiovascular disease were examined in the SMART study. ${ }^{29}$ This large, international, multicenter trial was designed to determine which of two different HIV treatment strategies, continuous viral suppression or drug conservation, would result in greater overall clinical benefit. In the drug conservation group, HAART interrupted until CD4+ cell count fell below 250 cells $/ \mathrm{mm}^{3}$, then restarted and continued until the CD4 was above 350 cells $/ \mathrm{mm}^{3}$. Over 5400 subjects were enrolled at 318 clinical sites in 33 countries over 4 years. Randomization was halted in January 2006 when the data safety monitoring board reported an increased relative risk of several adverse events in the drug conservation group. Further analyses showed significant increases in the risk of death, disease progression, myocardial infarction, as well as non-AIDS events. Other studies demonstrated that the discontinuation of HAART was associated with adverse effects in many surrogate markers for cardiovascular disease, including circulating levels of HDL cholesterol, D-dimer, interleukin-6, and other biomarkers, ${ }^{30}$ highlighting the role of infection and inflammation in promoting atherosclerosis and other clinical complications.

It is important to note that the SMART study was performed between 2002 and 2006. Progress in drug 
development has led to the development of many treatment regimens that are relatively free of metabolic toxicities, so the decisions on treatment switching are limited to ones with prior drug toxicity or few remaining treatment options. Of course, other therapies like weight control and smoking cessation must be stressed in all cases as they likely have a greater influence on outcome than do individual antiretroviral agents.

Many randomized clinical trials have been performed comparing the body composition and metabolic effects of different antiretroviral combinations in the management of previously antiretroviral-naïve subjects. In these studies, NRTIs, PIs, and NNRTIs have been randomized on a fixed backbone, while current and future studies will evaluate entry and integrase inhibitors in a similar fashion. In addition, class-sparing regimens have been evaluated. In general, studies have shown that newer generations of NRTIs are highly effective in avoiding the development of lipoatrophy, newer generations of PIs are highly effective in avoiding the development of dyslipidemia and insulin resistance, and the newer classes of antiretroviral agents are free of serious metabolic toxicities.

Most early studies involved a switch from PI- to NNRTIbased HAART, without a change in nucleoside therapy and with variable benefits related to the specific therapies being stopped and started. ${ }^{41}$ However, no changes in body composition were seen in these studies. Other studies, evaluating a switch from thymidine analog NRTI to other agents (eg, tenofovir or abacavir) led to a mild increase in limb fat and SAT, as well as to metabolic improvements. Other treatment switches have been performed looking at the effects of newer classes of agents (ie, integrase inhibitors), as well as studies designed to evaluate treatment simplification strategies. In general, the effect size for preventing lipoatrophy is greater than the effect size seen after switching treatment, suggesting a degree of irreversibility in the damage.

\section{Treatment of dyslipidemia}

Standard dietary modifications have only a modest effect on serum lipid concentrations, though a few studies showed that standard dietary advice led to prevention of weight gain and less dyslipidemia than no intervention. ${ }^{42,43}$ Several studies have shown that the fibrate subclass of antihyperlipidemia medications decreases serum triglyceride concentrations but does not significantly affect serum cholesterol concentrations. In contrast, HMG-CoA reductase inhibitors (statins) decrease serum cholesterol concentrations and, to a lesser extent, triglyceride concentrations. Despite these effective agents, the goals of clinical management of hyperlipidemia, as defined by National Cholesterol Education Program criteria, are often not reached in HIV-infected patients. ${ }^{42}$ It is important to remember that metabolism of some hypolipidemic medications is affected by ritonavir, through inhibition of cytochrome-mediated metabolism (CYP3A4). Consequently, pravastatin and rosuvastatin have become the drugs of choice for the treatment of dyslipidemia as they lack this specific drug-drug interaction, though they are not necessarily free of all interactions.

\section{Treatment of insulin resistance and diabetes mellitus}

The management of insulin resistance and diabetes mellitus should be the same in HIV-infected and noninfected patients, with the exception of possible antiviral regimen change. There is no official guideline for the pharmacologic management of insulin resistance in HIV- or non-HIV-infected individuals. Diabetes mellitus in HIV-infected individuals may be either ketosis prone or ketosis resistant, although the latter is the more common. Diet and oral agents are usually adequate to successfully manage most of the patients with this syndrome.

\section{Treatment of lipoatrophy}

Of all of the treatment-associated side effects, lipoatrophy has received the most attention. The most important advance in its management was the realization of the role played by thymidine analog NRTIs. Studies demonstrating a lack of developing lipoatrophy with alternative therapies led to the strategic avoidance of the problem. ${ }^{20,21}$ The situation in patients with clinically detectable lipoatrophy is less promising, as drug switching leads to only partial reversal of lipoatrophy. It has been suggested that the duration of thymidine analog therapy may determine if and how much reversibility is possible. Studies have also shown that depletion in mitochondrial DNA and mitochondrial dysfunction do not reverse completely, even long after thymidine analog therapy has been discontinued. Several agents, such as thiazolidinediones and uridine, have shown biologic activity in SAT and promote relatively minor amounts of reversal of lipoatrophy; however, there are no strong recommendations or guidelines support for such treatments. Several forms of plastic surgery have been applied to patients with facial lipoatrophy, with aesthetic success for several types of fillers; ${ }^{44}$ other therapies, including implants, have been used anecdotally in other body sites. The major drawback of plastic surgery is the lack of insurance coverage in many needy patients. 


\section{Treatment of lipohypertrophy}

Two well-known factors that affect total body and regional fat mass in non-HIV-infected subjects are diet and exercise. There has been relatively little study of these lifestyle changes in HIV-infected individuals. Both resistance exercise and a moderate exercise program were shown to reduce waist circumference and/or truncal fat content. A study of hypocaloric feeding and combined resistance and aerobic exercise in obese, HIV-infected women led to a significant loss of body weight and some VAT; however, there was no improvement of metabolic alterations, either insulin sensitivity or serum lipid concentrations. ${ }^{45}$ As these studies were performed relatively early in the HAART era, it was speculated that mitochondrial dysfunction related to thymidine analog therapy prevented the metabolic improvement. Studies performed in subjects not receiving thymidine analogs have shown benefits of diet and exercise as well as insulin-sensitizing agents. ${ }^{46}$

Human growth hormone has been shown to significantly reduce VAT in non-HIV-infected men with truncal obesity. ${ }^{47}$ Similar effects on VAT were seen in studies of HIV-infected subjects, ${ }^{48}$ although the drug did not gain FDA approval for that indication. However, a growth hormone-releasing factor has also been shown to significantly decrease VAT $^{49}$ and the FDA did approve the drug for that indication. As opposed to growth hormone, therapy is metabolically neutral. Limits in the effect size, cost, and need for daily self-injection will be major limiting factors in its application.

\section{Conclusion}

The development and application of HAART has had a profound effect on the natural course of HIV infection in the developed world and, increasingly, in the developing world. The recognition of adverse outcomes other than those related to immune deficiency has broadened the tasks required of the HIV caregiver to include primary care, especially in preventive cardiology. Unraveling the secrets surrounding HIV-associated lipodystrophy has provided important lessons about the interaction among infection, inflammation, and metabolic dysregulation.

\section{Disclosure}

The authors report no conflicts of interest in this work.

\section{References}

1. Garg A. Acquired and inherited lipodystrophies. N Engl J Med. 2004; 350:1220-1234.

2. Safrin $\mathrm{S}$, Grunfeld C. Fat distribution and metabolic changes in patients with HIV infection. AIDS. 1999;13:2493-2505.

3. Palella FJ, Baker RK, Moorman AC, et al. Mortality in the highly active antiretroviral therapy era: changing causes of death and disease in the HIV outpatient study. J Acquir Immune Defic Syndr. 1998;43:27-34.
4. Carr A, Samaras K, Burton S, et al. A syndrome of peripheral lipodystrophy, hyperlipidemia and insulin resistance in patients receiving protease inhibitors. AIDS. 1998;12:F51-F58.

5. Podzamczer D, Ferrer E, Martínez E, et al. How much fat loss is needed for lipoatrophy to become clinically evident. AIDS Res Hum Retroviruses. 2009;25:563-567.

6. Vague J. The degree of masculine differentiation of obesities: a factor determining predisposition to diabetes, atherosclerosis, gout, and uric calculous disease. Am J Clin Nutr. 1956;4:20-23.

7. Bacchetti P, Gripshover B, Grunfeld C, et al. Fat distribution in men with HIV infection. J Acquir Immune Defic Syndr. 2005;40:121-131.

8. Study of fat redistribution and metabolic change in HIV infection (FRAM). Fat distribution in women with HIV infection. $J$ Acquir Immune Defic Syndr. 2006;42:562-571.

9. Brown TT, Chu H, Wang Z, et al. Longitudinal increases in waist circumference are associated with HIV-serostatus, independent of antiretroviral therapy. AIDS. 2007;21:1731-1738.

10. Grunfeld C, Pang M, Doerrler W, et al. Lipids, lipoproteins, triglyceride clearance, and cytokines in human immunodeficiency virus infection and the acquired immunodeficiency syndrome. J Clin Endocrinol Metab. 1992;74:1045-1052.

11. Grunfeld C, Kotler DP, Shigenaga JK, et al. Circulating interferon- $\alpha$ levels and hypertriglyceridemia in the acquired immunodeficiency syndrome. Am J Med. 1991;90:154-162.

12. Lumpkin MM. FDA public health advisory: reports of diabetes and hyperglycemia in patients receiving protease inhibitors for the treatment of human immunodeficiency virus (HIV). Rockville, MD: US Food and Drug Administration; June 11, 1997.

13. Purnell JQ, Zambon A, Knopp RH, et al. Effect of ritonavir on lipids and post-heparin lipase activities in normal subjects. AIDS. 2000; $14: 51-57$.

14. Noor MA, Lo JC, Mulligan K, et al. Metabolic effects of indinavir in healthy HIV-seronegative men. AIDS. 2001;15:F11-F18.

15. Maia BS, Engelson ES, Wang J, Kotler DP. Antiretroviral therapy affects the composition of weight loss in HIV infection: implications for clinical nutrition. Clin Nutr. 2005;24:971-978.

16. Riddler SA, Smit E, Cole SR, et al. Impact of HIV infection and HAART on serum lipids in men. JAMA. 2003;289:2978-2982.

17. Murata H, Hruz PW, Mueckler M. The mechanism of insulin resistance caused by HIV protease inhibitor therapy. J Biol Chem. 2000; 275:20251-20254.

18. Liang JS, Distler O, Cooper DA, et al. HIV protease inhibitors protect apolipoprotein $\mathrm{B}$ from degradation by the proteosome: a potential mechanism for protease inhibitor-induced hyperlipidemia. Nat Med. 2001;7:1327-1331.

19. McKenzie R, Fried MW, Sallie R, et al. Hepatic failure and lactic acidosis due to fialuridine (FIAU), an investigational nucleoside analogue for chronic hepatitis B. N Engl J Med. 1995;333:1099-1105.

20. Gallant JE, Staszewski S, Pozniak AL, et al. Efficacy and safety of tenofovir DF vs stavudine in combination therapy in antiretroviral-naive patients: a 3-year randomized trial. JAMA. 2004;292:191-201.

21. Gallant JE, DeJesus E, Arribas JR, et al. Tenofovir DF, emtricitabine, and efavirenz vs zidovudine, lamivudine, and efavirenz for HIV. N Engl J Med. 2006;354:251-260.

22. Blumer RM, van Vonderen MG, Sutinen J, et al. Zidovudine/lamivudine contributes to insulin resistance within 3 months of starting combination antiretroviral therapy. AIDS. 2008;22:227-236.

23. Fleischman A, Johnson S, Systrom DM, et al. Effects of a nucleoside reverse transcriptase inhibitor, stavudine, on glucose disposal and mitochondrial function in muscle of healthy adults. Am J Physiol Endocrinol Metab. 2007;292:E1666-E1673.

24. Martin A, Smith SE, Carr A, et al. Reversibility of lipoatrophy in HIVinfected patients 2 years after switching from a thymidine analogue to abacavir: the MITOX Extension Study. AIDS. 2004;18:1029-1036.

25. Franssen R, Sankatsing RR, Hassink E, et al. Nevirapine increases high-density lipoprotein cholesterol concentration by stimulation of apolipoprotein A-1 production. Arterioscler Thromb Vasc Biol. 2009; 29:1336-1341. 
26. Ranade K, Geese WJ, Noor M, et al. Genetic analysis implicates resistin in HIV lipodystrophy. AIDS. 2008;22:1561-1568.

27. Ross R. Atherosclerosis: an inflammatory disease. $N$ Engl J Med. 1999;340:115-126.

28. Deeks SG. HIV infection, inflammation, immunosenescense, and aging. Annu Rev Med. 2011;62:141-155.

29. El Sadr WM, Lundgren JD, Neaton JD, et al. CD4+ count-guided interruption of antiretroviral treatment. N Engl J Med. 2006;355:2283-2296.

30. Kuller LH, Tracy R, Belloso W, et al. Inflammatory and coagulation biomarkers and mortality in patients with HIV infection. PLoS Med. 2008; 5:e203.

31. Calmy A, Gayet-Ageron A, Montecucco F, et al. HIV increases markers of cardiovascular risk: results from a randomized, treatment interruption trial. AIDS. 2009;23:929-939.

32. Torriani F, Komarow L, Parker RA, et al. Endothelial function in human immunodeficiency virus-infected antiretroviral-naive subjects before and after starting potent antiretroviral therapy: the ACTG (AIDS Clinical Trials Group) Study 5152s. J Am Coll Cardiol. 2008;52: 569-576

33. Marin A, Casado JL, Aranzabal L, et al. Validation of a specific questionnaire on psychological and social repercussions of the lipodystrophy syndrome in HIV-infected patients. Qual Life Res. 2006;15:767-775.

34. Ammassari A, Murri R, Pezzotti P, et al. Self-reported symptoms and medication side effects influence adherence to highly active antiretroviral therapy in persons with HIV infection. J Acquir Immune Defic Syndr. 2001;28:445-449.

35. Friis-Moller N, Sabin CA, Weber R, et al. Combination antiretroviral therapy and the risk of myocardial infarction. $N$ Engl J Med. 2003;349:1993-2003.

36. Klein D, Hurley LB, Quesenberry CP Jr, Sidney S. Do protease inhibitors increase the risk for coronary heart disease in patients with HIV infection? J Acquir Immune Defic Syndr. 2002;30:471-477.

37. Law MG, Friis-Moller N, El-Sadr WM, et al. The use of the Framingham equation to predict myocardial infarctions in HIV-infected patients: comparison with observed events in the D:A:D Study. HIV Med. 2006; 7:218-230

38. Carr A, Emery S, Law M, et al. An objective case definition of lipodystrophy in HIV-infected adults: a case-control study. Lancet. 2003; 361:726-735.

39. Schambelan M, Benson CA, Carr J, et al. Management of metabolic complications associated with antiretroviral therapy for HIV-1 infection: recommendations of an International AIDS Society-USA panel. J Acquir Immune Defic Syndr. 2002;31:257-275.
40. Wohl DA, McComsey G, Tebas P, et al. Current concepts in the diagnosis and management of metabolic complications of HIV infection and its therapy. Clin Infect Dis. 2006;43:645-653.

41. Martínez E, Arnaiz JA, Podzamczer D, et al. Substitution of nevirapine, efavirenz, or abacavir for protease. Inhibitors in patients with human immunodeficiency virus infection. $N$ Engl J Med. 2003; 349:1036-1046.

42. Lazzaretti R, Pinto-Ribeiro J, Kummer R, Polanczyk C, Sprinz E. Dietary intervention when starting HAART prevents the increase in lipids independently of drug regimen: a randomized trial [Abstract WEAB303]. Oral abstract session: 4th IAS Conference on HIV Pathogenesis, Treatment and Prevention; 2007 Jul 22-25; Sydney, Australia.

43. Joy T, Keough HM, Hadigan C, et al. Dietary fat intake and relationship to serum lipid levels among HIV-infected subjects with metabolic abnormalities in the era of HAART. AIDS. 2007;21:1591-1600.

44. Valantin MA, Aubron-Olivier C, Ghosn J, et al. Polylactic acid implants (New-Fill) to correct facial lipoatrophy in HIV-infected patients: results of the open-label study VEGA. AIDS. 2003;17:2471-2477.

45. Engelson ES, Agin D, Werber-Zion G, et al. Effects of a diet and exercise weight loss regimen on obese, HIV-infected women. Metabolism. 2006;55:1327-1336.

46. Yarasheski KE, Cade WT, Overton ET, et al. Exercise training augments the peripheral insulin-sensitizing effects of pioglitazone in HIV-infected adults with insulin resistance and central obesity. Am J Physiol Endocrinol Metab. 2011;300:E243-E251.

47. Johannsson G, Marin P, Lonn L, et al. Growth hormone treatment of abdominally obese men reduces abdominal fat mass, improves glucose and lipoprotein metabolism, and reduces diastolic blood pressure. JClin Endocrinol Metab. 1997;82:727-734.

48. Kotler DP, Muurahainen N, Grunfeld C, et al. Effects of growth hormone on abnormal visceral adipose tissue accumulation and dyslipidemia in HIV-infected patients. J Acquir Immune Defic Syndr. 2004;35:239-254.

49. Falutz J, Allas S, Blot K, et al. Effects of a growth hormone releasing factor analogue (growth hormone releasing hormone) on body composition and metabolic indices in HIV-infected patients. N Engl J Med. 2007;357:2359-2370.
HIV/AIDS - Research and Palliative Care

\section{Publish your work in this journal}

HIV/AIDS - Research and Palliative Care is an international, peerreviewed open-access journal focusing on advances in research in HIV, its clinical progression and management options including antivira treatment, palliative care and public healthcare policies to control viral spread. The journal welcomes original research, basic science,

\section{Dovepress}

clinical \& epidemiological studies, reviews \& evaluations, expert opinion \& commentary, case reports \& extended reports. The manuscript management system is completely online and includes a very quick and fair peer-review system. Visit http://www.dovepress.com/ testimonials.php to read real quotes from published authors. 